\title{
METALLICITY STRUCTURES OF THE MILKY WAY
}

\author{
ROLAND BUSER \\ Astronomical Institute, University of Basel, Switzerland
}

AND

JIANXIANG RONG

Astronomy Department, Nanjing University, China

\begin{abstract}
The metallicity-sensitive $(U-G)$ colors from the new homogeneous catalog of photographic RGU data in seven high-latitude fields have been used to determine the larger-scale metallicity distributions of the Galactic population components. For the thick disk, preliminary analysis based on our best structural models provides a mean metallicity $\langle[M / H]\rangle=$ $-0.6 \pm 0.3 d e x$ and a marginal vertical metallicity gradient $\approx-0.1 d e x / k p c$. The observed color distributions are further consistent with the (old) thin disk having mean abundance $\langle[M / H]\rangle=-0.3 \pm 0.2 d e x$ and abundance gradient $\partial[M / H] / \partial z=-0.6$ dex $/ k p c$.
\end{abstract}

Based on the results obtained for the structural parameters [1], we have determined the metallicity structures of the different population components in a complementary analysis of the new RGU catalog [2]. In particular, parameter variation is confined to their optimized values and to within the constraints imposed by the relevant $\chi^{2}$-curves and frequency distributions derived from the $(G, G-R)$ data. Model star count and color distributions are then calculated allowing for appropriate ranges of mean metallicities and vertical metallicity gradients for the different components and using the corresponding metallicity-dependent changes in the $(G, G-R)$ and $(G, U-G)$ color-magnitude calibrations [3]. Finally, the $\chi^{2}$-analysis is performed by requiring the models to also provide good matches to the observed distributions in the metal-sensitive $(U-G)$ color, $N(U-G)$. Evaluation of the globally lowest- $\chi^{2}$-models then leads to the determination of the optimum values for the mean metallicities, $\langle[M / H]\rangle$, and metallicity gradients, $\partial[M / H] / \partial z$, of the Galactic population components. 
TABLE 1. Mean metallicity and vertical metallicity gradient for thick disk

\begin{tabular}{lccccccc}
\hline Field & $l$ & $b$ & Area $\left(\right.$ deg $\left.^{2}\right)$ & $m_{G, l i m}$ & No.** & $\langle[M / H]\rangle$ & $\partial[M / H] / \partial z$ \\
\hline Praesepe & 205.9 & 32.4 & 3.56 & 15.0 & 1228 & -0.81 & -0.20 \\
M 101 & 101.0 & 60.0 & 2.00 & 18.5 & 1388 & -0.60 & -0.04 \\
M 67 & 210.6 & 32.2 & 1.70 & 16.0 & 748 & -0.78 & -0.20 \\
SA 54 & 200.1 & 58.8 & 2.56 & 18.5 & 1359 & -0.52 & -0.05 \\
SA 57 & 65.5 & 85.5 & 2.61 & 19.0 & 1807 & -0.49 & -0.03 \\
SA 141 & 245.0 & -85.8 & 1.92 & 17.5 & 758 & -0.76 & -0.15 \\
M 5 & 4.0 & 47.0 & 1.05 & 18.5 & 1819 & -0.54 & -0.03 \\
Combined & & & 15.40 & & 9107 & -0.58 & -0.10 \\
\hline
\end{tabular}

Table 1 gives a summary of the field data and the optimized values obtained for the mean metallicities and vertical metallicity gradients of the thick disk as derived from the $(U-G)$ color distributions in each individual field, plus the weighted mean for the combined survey. The present analysis suggests that the thick disk has mean metallicity $\langle[M / H]\rangle=-0.6 \operatorname{dex}$ (with dispersion $\sigma_{\langle[M / H]\rangle}=0.3$ dex $)$ and only a marginal metallicity gradient $\partial[M / H] / \partial z=-0.1$ dex $/ k p c$.

For the thin disk, we find a relatively low optimized value of $\langle[M / H]\rangle=$ $-0.3 \pm 0.2 d e x$, along with a vertical gradient of $\partial[M / H] / \partial z=-0.6 d e x / k p c$. No gradient, however, has been found for the halo $(\langle[M / H]\rangle=-1.5 \pm$ $0.5 d e x)$, which, due to the relatively bright magnitude limit, is undersampled in the present survey.

These results are in good agreement with recent evidence from detailed studies of kinematic and chemical data for more local stellar samples. In particular, they corroborate and extend to the larger-scale Galaxy the conclusions drawn from photoelectric Walraven photometry for a small complete sample of the brighter SGP stars [4], and thus will help further constrain current models of the formation and early evolution of the Galaxy [5].

\section{References}

1. Buser, R. and Rong, J.X. 1994, this volume

2. Buser, R., Karaali, S., Topaktas, L., Karatas, Y. 1994, A\&AS (in press)

3. Buser, R. and Fenkart, R.P. 1990, A\&A 239, 243

4. Trefzger, Ch.F., Pel, J.W., Gabi, S. 1994, A\&A (in press)

5. Majewski, S.R. 1993, ARA\&A 31, 575 